\title{
Identification of Nonlinear Lateral Flow Immunoassay State Space Models via Particle Filter Approach
}

\author{
Nianyin Zeng, Zidong Wang, Yurong Li, Min Du and Xiaohui Liu
}

\begin{abstract}
In this paper, the particle filtering approach is used, together with the kernel smoothing method, to identify the state space model for the lateral flow immunoassay through available but short time-series measurement. The lateral flow immunoassay model is viewed as a nonlinear dynamic stochastic model consisting of the equations for the biochemical reaction system as well as the measurement output. The renowned extended Kalman filter is chosen as the importance density of the particle filter for the purpose of modeling the nonlinear lateral flow immunoassay. By using the developed particle filter, both the states and parameters of the nonlinear state-space model can be identified simultaneously. The identified model is of fundamental significance for the development of lateral flow immunoassay quantification. It is shown that the proposed particle filtering approach works well for modeling the lateral flow immunoassay.
\end{abstract}

Index Terms-Lateral flow immunoassay; particle filter; extended Kalman filter; state estimation; parameter estimation.

\section{INTRODUCTION}

$\mathbf{T}$ HE lateral flow immunoassay (LFIA), which utilizes the specific interaction between antigens and antibodies, has been extensively studied in the general area of biomedical engineering and widely applied to a variety of point-of-care fields over the past decades, [3], [9], [11], [12], [14], [34] and the references therein. The LFIA has been gaining particular research attention due primarily to its attractive properties such as short analysis time, ease of use, low cost, high sensitivity, good specificity and satisfactory stability [28], [33]. Recently, in addition to the traditional trend of physically improving the biochemical properties of the strips (see e.g. [1], [3], [9], [12]), there has been a growing research interest in establishing a mathematical model of LFIA so as to develop the quantitative

Copyright (c) 2011 IEEE. Personal use of this material is permitted. However, permission to use this material for any other purposes must be obtained from the IEEE by sending a request to pubs-permissions@ieee.org.

This work was supported in part by the International Science and Technology Cooperation Project of China under Grant 2009DFA32050, Natural Science Foundation of China under Grants 61104041, International Science and Technology Cooperation Project of Fujian Province of China under Grant 2009 I0016.

N. Zeng is with the College of Electrical Engineering and Automation, Fuzhou University, Fuzhou 350002, P. R. China, and also with the Fujian Key Laboratory of Medical Instrumentation and Pharmaceutical Technology, Fuzhou 350002, P. R. China. (e-mail: nianyin.zeng@gmail.com).

Z. Wang and X. Liu are with the Department of Information Systems and Computing, Brunel University, Uxbridge, Middlesex, UB8 3PH, United Kingdom. (e-mail: Zidong.Wang@brunel.ac.uk).

Y. Li and M. Du are with the Fujian Key Laboratory of Medical Instrumentation and Pharmaceutical Technology, Fuzhou University, Fuzhou 350002, P. R. China. instruments (see e.g. [4], [6], [8], [11], [14]). It has been revealed that the mathematical model plays a very important role when producing strips with high-sensitivity and low constant of variance for the purpose of quantification. Especially, an adequate mathematical model allows us to predict kinetic characteristics and test the effects of various design parameters in a both rapid and inexpensive way. Furthermore, such a model could also enable us to optimize device performance [26], [27], [34]. Therefore, it is obvious that the progress in mathematical modeling is crucial for facilitating the usage of the lateral flow immunoassay.

Let us now provide a brief review of latest literature on the modeling issue of the LFIA. In [26], [27], the convection diffusion reaction equations have been used to model the lateral flow immunoassay systems and the simulation has been carried out by using the COMSOL software. Recently, a nonlinear dynamic stochastic model has been considered in [34] for sandwich-type lateral flow immunoassay where the established model is composed of the biochemical reaction system equations and the observation equation. In [34], the system state equations describe the dynamics of the concentration distribution subject to stochastic disturbances, and the system measurements are determined in terms of an observation equation containing measurement noises. The extend Kalman filter (EKF) has been designed in [34] which serves as a suboptimal filter for identifying both the states and parameters of the nonlinear state-space model. The EKF algorithm linearizes the nonlinear model by Taylor expansion and then uses the traditional Kalman filter for the linearized model. Because of its versatility and effectiveness, the EKF algorithm has been proven to perform well under the assumptions that the nonlinearity is linearizable and the noise is Gaussian. Unfortunately, when the models are highly nonlinear and/or noise disturbances are non-Gaussian distributed, the EKF algorithm may behave pretty poorly. In the context of modeling the lateral flow immunoassay, the model to be established inherently involves high nonlinearities, and there is a great need to seek a more appropriate modeling approach that is capable of handling both the nonlinearities and the nonGaussian noises. In search of such a candidate, the particle filter appears to be an ideal one that can be applied to more general systems than the traditional Kalman filtering methods [5], [10].

Basically, the particle filter is a recursive Bayesian filter by Monte Carlo simulations. In the past decade, particle filters have gained much attention with wide applications in 
state estimation problems for nonlinear and/or non-Gaussian system, see e.g. [5], [7], [10], [13]. It is remarkable that particle filters represent the posterior density $p\left(x_{k} \mid z_{1: k}\right)$ by a set of random samples (also called particles) with associated weights $\left\{\left(x_{k}^{i}, \omega_{k}^{i}\right), i=1, \ldots, N\right\}$. Thus, it can effectively and exactly represent the required posterior density function as the number of particles becomes very large. Accordingly, the particle filter has the great advantage of being able to deal with any functional nonlinearity and noise distribution. However, despite its great potential in modeling biomedical systems, so far, there have been very few results in existing literature with respect to the application of particle filters in jointly identifying the system states and parameters of the lateral flow immunoassay state-space model via short but available time series data. It is, therefore, our intention in this paper to fill the gap by investigating the use of particle filters in the presence of nonlinearities and short time series.

Applying the particle filtering approach in identifying LFIA state-space model is a non-trivial task. There are several challenging issues that typically emerge for modelling LFIAs. Apart from the high nonlinearities, both the states and parameters of the LFIA should be identified simultaneously. Furthermore, a LFIA exhibits a distinct feature that there are only few observations (measurement outputs). That is, the measurements of a LFIA are in the form of high-resolution images, and the number of consecutive images (time series) is usually small because of the physical constraints (e.g. instrument limit and experiment cost). To date, much effort has been devoted to identify the unknown parameters in a general nonlinear model by using particle filters. One of the common strategies is to add the parameters as part of the state space vector which, however, would easily lead to the particle degeneration and hence inaccurate estimation [5], [15]. In [16], a dual particle filter has been proposed that use two particle filters to estimate states and parameters of a nonlinear system, where such a dual filter can improve the accuracy but suffer from significant increases of running time and computation burden. In this paper, the kernel smoothing method developed in [2], [5] will be employed to avoid the degeneration problem and also handle both the fixed and time-varying parameters conveniently by tuning the smoothing factor.

In this paper, we aim to develop a particle filtering algorithm in combination with the kernel smoothing method in order to jointly estimate system parameters and states of the lateral flow immunoassay model through available but short timeseries measurement. The identified lateral flow immunoassay model is proven to be more accurate than the one obtained from the traditional EKF algorithm. The main contribution of this paper is mainly twofold. 1) The particle filter equipped with the kernel smoothing method is, for the first time, applied to model the lateral flow immunoassay system. The developed algorithm represents the first of few ones that are capable of simultaneously identifying the system parameters and actual concentration distribution of the lateral flow immunoassay through an iterative procedure by using a small number of observations. 2) It is shown that the proposed particle filtering approach works well for modeling the lateral flow immunoassay.
The rest of this paper is organized as follows. In Section II, the nonlinear state-space model for the lateral flow immunoassay system is introduced. The particle filters combining with the kernel smoothing method for jointly estimating system parameters and states are described in Section III. In Section IV, our developed algorithm is applied to the lateral flow immunoassay system and the results of joint parameter and state estimation by the algorithm are discussed. Finally, concluding remarks are given in Section V.

\section{The Lateral Flow Immunoassay Model AND PROBLEM FORMULATION}

A typical configuration of lateral flow immunoassay, as shown in Fig. 1 [24], consists of a variety of materials such as sample pad, nitrocellulose membrane, conjugate pad and wicking pad. The primary antibodies are immobilized within a defined detection zone (test line) on the membrane. The secondary antibodies are conjugated with reporter particles such as colloidal gold, carbon black, fluorescent, or paramagnetic monodisperse latex particle [23].

In this paper, we focus on the sandwich format of gold immunochromatographic strip where the reporter particle uses the colloidal gold nanoparticles. With the presence of an antigen in the sample, a sandwich-type assay is formed between the secondary antibody-immobilized gold nanoparticle immunocomplex and the primary antibody immobilized on the membrane. After the antigen-antibody reaction, the red color caused by the accumulation of gold nanoparticle at that location would appear on the membrane [23], [28]. The color intensity of the red test line (signal intensity), which relates directly to the concentration of the target protein in the standard or spiked samples, is assessed visually or by a reader system for quantitative analysis [1].

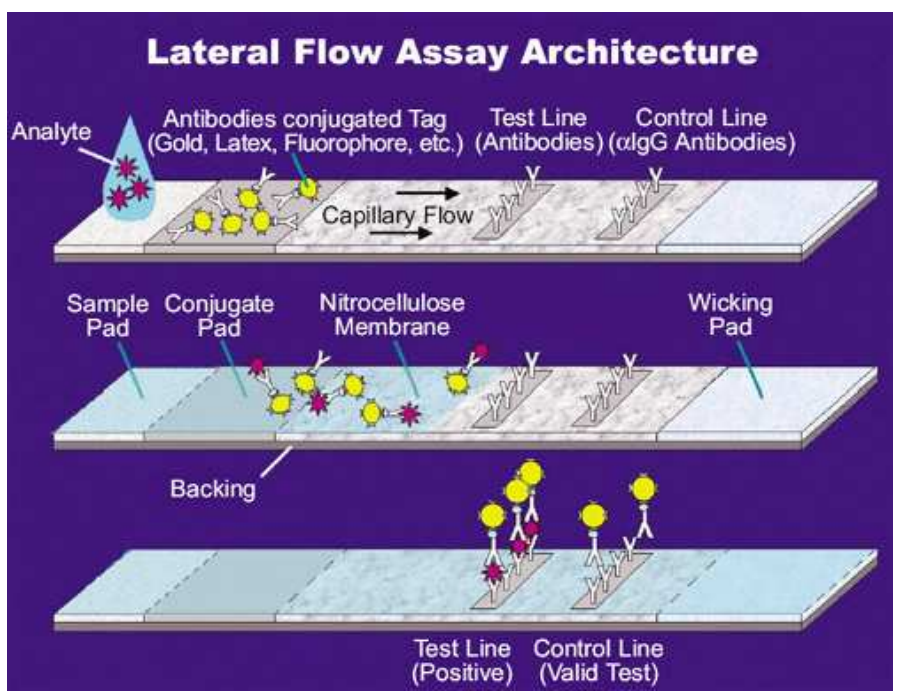

Fig. 1. Lateral flow immunoassay architecture.

In this paper, for simplicity, we only consider a single target analyte in the sample. Thus, the biochemical reactions of the lateral flow immunoassay signal pathway without consideration of the control line can be generally summarized as follows [26]: 
1) Assume that the sample contains a target analyte $A$. When the sample migrates through the conjugate pad, the analyte interacts with the particulate color particle conjugate $P$ to form particle-analyte complexes $P A$,

$$
A+P \underset{k_{2}}{\stackrel{k_{1}}{\rightleftharpoons}} P A
$$

2) The free analyte in the sample and the particle-analyte complexes both migrate into the membrane by the capillary action. Free analyte of type $i(A)$ and particleanalyte complexes $P A$ interact with the immobilized ligands of type $i(R)$ to form the complexes,

$$
\begin{gathered}
A+R \underset{k_{4}}{\stackrel{k_{3}}{\rightleftharpoons}} R A \\
P A+R \underset{k_{6}}{\stackrel{k_{5}}{\rightleftharpoons}} R P A
\end{gathered}
$$

3) Additionally, unbound particulate conjugate $P$ may bind to the complex $R A$ to form the complex $R P A$,

$$
P+R A \underset{k_{8}}{\stackrel{k_{7}}{\rightleftharpoons}} R P A
$$

Let $x_{1}, x_{2}, x_{3}, x_{4}, x_{5}$ and $x_{6}$ be the concentration of $A, P, P A, R, R A$ and $R P A$, respectively. For demonstration purpose, it is assumed that there is no time-delay between the biochemical reactions (1)-(4). The rates of the reactions are defined as follows:

$$
\begin{aligned}
& v_{1}=k_{1} x_{1} x_{2}-k_{2} x_{3} \\
& v_{2}=k_{3} x_{1} x_{4}-k_{4} x_{5} \\
& v_{3}=k_{5} x_{3} x_{4}-k_{6} x_{6} \\
& v_{4}=k_{7} x_{2} x_{5}-k_{8} x_{6}
\end{aligned}
$$

where $k_{1}, k_{3}, k_{5}, k_{7}$ and $k_{2}, k_{4}, k_{6}, k_{8}$ are the association and dissociation rate constants, respectively. The stoichiometrix for the biochemical reaction of the lateral flow immunoassay is given by

$$
S=\left[\begin{array}{cccc}
-1 & -1 & 0 & 0 \\
-1 & 0 & 0 & -1 \\
1 & 0 & -1 & 0 \\
0 & -1 & -1 & 0 \\
0 & 1 & 0 & -1 \\
0 & 0 & 1 & 1
\end{array}\right]
$$

Let $x=\left[x_{1}, x_{2}, \ldots, x_{6}\right]^{T}, V=\left[v_{1}, v_{2}, \ldots, v_{4}\right]^{T}$, and $\theta=$ $\left[k_{1}, k_{2}, \ldots, k_{9}\right]^{T}$ to denote the parameters to be estimated, which are the association and dissociation rate constants in the vector $V(x(k))$. The nonlinear state-space model of the lateral flow immunoassay can be given as follows [34]:

$$
\begin{aligned}
x(k+1) & =f(x(k), \theta)+w(k) \\
z(k) & =g(x(k), \theta)+v(k)
\end{aligned}
$$

where $x(k)$ is the vector of state variables which are concentrations of antibodies, antigens or complex material; $z(k)$ is the measurement data from experiments at the time point $k ; f(x(k), \theta)=S V(x(k))$ with $S$ being a stoichiometric matrix that describes the biochemical transformation in a biochemical network and $V(x(k))$ being the vector of reaction rates (usually the vector of nonlinear function of the state) [30], $g(x(k), \theta)=k_{9}\left(x_{3}+x_{6}\right)$ is the measurement model function with $\theta$ being a parameter vector to be identified; $w(k)$ and $v(k)$ denote the zero-mean uncorrelated Gaussian noises with covariance matrices $Q_{k}$ and $R_{k}$, respectively.

The main purpose of this paper is to jointly estimate the parameters and states of the model (9)-(10) via the particle filter combining with kernel smoothing method from the possibly small number of the measured data.

\section{Kernel-Smoothing-BASEd Particle Filtering FOR STATES AND PARAMETERS ESTIMATION}

In this section, we introduce the particle filters theory as well as the kernel smoothing method for estimating system states and parameters. For more details we refer the readers to [5], [10], [18] and the reference therein.

\section{A. Particle Filters}

The particle filter is essentially a recursive Bayesian filter based on Monte Carlo simulations. Particle filters are capable of dealing with nonlinear and/or non-Gaussian systems in a convenient yet efficient way. Consider the following nonlinear state space system described by state transition and measurement equations:

$$
\begin{aligned}
& x_{k}=f\left(x_{k-1}, w_{k-1}\right) \\
& z_{k}=h\left(x_{k}, v_{k}\right)
\end{aligned}
$$

where $k$ is a non-negative integer, $x_{k} \in \mathbb{R}^{n}$ is the system state vector, $z_{k} \in \mathbb{R}^{r}$ is the observation vector (measurement output), and $w_{k}$ and $v_{k}$ are independently and identically distributed noises for the process and measurements, respectively. Here, $f: \mathbb{R}^{n} \rightarrow \mathbb{R}^{n}$ is a nonlinear state transition function and $h: \mathbb{R}^{n} \rightarrow \mathbb{R}^{r}$ is a nonlinear measurement function.

From a Bayesian perspective, the aim of the state estimation is to infer the probability function $p\left(x_{k} \mid z_{1: k}\right)$ of the state $x_{k}$ given the sequence of all available measurements $z_{1: k}=\left\{z_{1}, z_{2}, \ldots, z_{k}\right\}$. Assuming that the initial condition $p\left(x_{0} \mid z_{0}\right) \equiv p\left(x_{0}\right)$ and $p\left(x_{k-1} \mid z_{1: k-1}\right)$ at time $k-1$ are available, the Bayesian estimation infers the posterior density function $p\left(x_{k} \mid z_{1: k}\right)$ in a recursive manner by two steps. Using the transition density one can perform the prediction step:

$$
p\left(x_{k} \mid z_{1: k-1}\right)=\int p\left(x_{k} \mid x_{k-1}\right) p\left(x_{k-1} \mid z_{1: k-1}\right) d x_{k-1}
$$

and update step uses the measurement $z_{k}$ to update the predicted density:

$$
p\left(x_{k} \mid z_{1: k}\right)=\frac{p\left(z_{k} \mid x_{k}\right) p\left(x_{k} \mid z_{1: k-1}\right)}{p\left(z_{k} \mid z_{1: k-1}\right)}
$$

where $p\left(z_{k} \mid z_{1: k-1}\right)$ is a normalizing factor independent of the state $x_{k}$.

In theory, the optimal solution to the state estimation problem can be derived by Bayesian filter via the recurrence relations (13)-(14) for the dynamic system. However, analytical solutions only exist in a restrictive set of cases when the noises are Gaussian distributed and the model is linear via the Kalman filtering or grid-based filtering methods. 
When these assumptions do not hold, it is almost impossible to determine the solutions analytically. As such, alternative approximate filters such as particle filters have been proposed in the literature to approximate the optimal Bayesian solution.

The key idea of particle filtering is to represent the posterior density $p\left(x_{k} \mid z_{1: k}\right)$ by a set of random samples (also called particles) with associated weights $\left\{\left(x_{k}^{i}, \omega_{k}^{i}\right), i=1, \ldots, N\right\}$ :

$$
p\left(x_{k} \mid z_{1: k}\right) \approx \sum_{i=1}^{N} \omega_{k}^{i} \delta\left(x_{k}-x_{k}^{i}\right)
$$

where $\sum_{i=1}^{N} \omega_{k}^{i}=1$, and $\delta(x)$ is Dirac's delta function that is equal to infinity if $x=0$ and otherwise zero. Thus, particle filters convert the integral operation into the sum formation to compute estimates based on the generated random samples from $p\left(x_{k} \mid z_{1: k}\right)$ and weights. However, direct sampling from the posterior density $p\left(x_{k} \mid z_{1: k}\right)$ is complex outside of a linear and Gaussian environment. The particle filter adopts a technique named importance sampling [17] to generate the random samples. The main idea of the importance sampling is to introduce a known probability density function $q\left(x_{k} \mid z_{1: k}\right)$, which is called importance density, from which samples $x_{k}^{i}$ can be drawn. As such, the importance weights are defined as

$$
\omega_{k}^{i} \propto \frac{p\left(x_{k}^{i} \mid z_{1: k}\right)}{q\left(x_{k}^{i} \mid z_{1: k}\right)}
$$

Letting the importance density be chosen to factorize such that

$$
q\left(x_{k} \mid z_{1: k}\right)=q\left(x_{0: k-1} \mid z_{1: k-1}\right) q\left(x_{k} \mid x_{0: k-1}, z_{1: k}\right),
$$

the recursive formula for the importance weights is obtained as follows

$$
\omega_{k}^{i} \propto \omega_{k-1}^{i} \frac{p\left(z_{k} \mid x_{k}^{i}\right) p\left(x_{k}^{i} \mid x_{k-1}^{i}\right)}{q\left(x_{k}^{i} \mid x_{k-1}^{i}, z_{k}\right)} .
$$

Therefore, one can obtain particles $x_{0: k}^{i}=\left\{x_{0: k-1}^{i}, x_{k}^{i}\right\}$ by augmenting each of the existing samples $x_{0: k-1}^{i} \sim$ $q\left(x_{0: k-1} \mid z_{1: k-1}\right)$ with the new states $x_{k}^{i} \sim q\left(x_{k} \mid x_{0: k-1}, z_{1: k}\right)$. With these particles and associated importance weights calculated by Eqn.(18), the estimated state vector, $\hat{x}_{k}$, is the mean of $p\left(x_{k} \mid z_{1: k}\right)$ and is calculated as

$$
\hat{x}_{k}=\sum_{i=1}^{N} \omega_{k}^{i} x_{k}^{i} \text {. }
$$

\section{B. Degeneracy Problem of Particle Filters}

The algorithm of particle filter described above has a degeneracy problem where, after a few iterations, the weight is only concentrated on a few particles (most particles have negligible weight). Thus, a large computational effort is devoted to updating particles whose contribution to the approximation to $p\left(x_{k} \mid z_{1: k}\right)$ is almost zero. Therefore, it is necessary to modify the algorithm to assure that degeneration of the particles will not take place. In this case, two additional practical techniques have been introduced: a) good choice of importance density and b) resampling method [10].

A good choice of importance density is critical to the performance of the particle filter method. Theoretically, the posterior distribution $p\left(x_{0: k} \mid z_{0: k}\right)$ would be the best option, but the calculations involved would be extremely difficult outside of a linear and Gaussian environment as mentioned above. Another approach is to use the prior distribution $p\left(x_{k} \mid x_{k-1}\right)$ with easy implementation, but it does not carry any information from new observations which would make the filter degenerates rapidly. Thus, to avoid these problems when using the posterior distribution and the prior distribution as the importance density, the suboptimal approximations to the optimal importance density by using extended Kalman filter are used in this paper as done in [18]. For this purpose, there is a need to state the Extended Kalman Filter (EKF) algorithm as follows [31]:

\section{Initialization}

For $k=0$, set

$$
\begin{aligned}
& \hat{x}(0 \mid 0)=E[x(0)]=x_{0}, \\
& P(0 \mid 0)=E\left[\left(x(0)-x_{0}\right)\left(x(0)-x_{0}\right)^{T}\right]=P_{x_{0}} .
\end{aligned}
$$

For $k=1,2,3, \ldots$ compute

Time update ('Predict')

State estimate time update: $\hat{x}(k \mid k-1)=f(\hat{x}(k-1 \mid k-1))$ Error covariance time update: $P(k \mid k-1)=\hat{A}(k-1) P(k-$ $1 \mid k-1) \hat{A}(k-1)^{T}+Q_{k-1}$

\section{Measurement update ('Correct')}

Compute the Kalman gain matrix: $K_{k}=P(k \mid k-$ 1) $\hat{C}(k)^{T}\left[\hat{C}(k) P(k \mid k-1) \hat{C}(k)^{T}+R_{k}\right]^{-1}$

Update the estimate with measurement $y(k): \hat{x}(k \mid k)=$ $\hat{x}(k \mid k-1)+K_{k}[y(k)-g(\hat{x}(k \mid k-1))]$

Error covariance measurement update: $P(k \mid k)=(I-$ $\left.K_{k} \hat{C}(k)\right) P(k \mid k-1)$.

The second method for reducing the degeneration phenomenon is to use the idea of resampling. The resampling approach has been used in [19] to eliminate particles with low weights and choose more particles in more probable regions. A new set $\left\{\hat{x}_{k}^{j}, j=1, \ldots, N\right\}$ is generated by sampling with replacement from an approximate discrete representation of $p\left(x_{0: t} \mid y_{1: t}\right)$ with probability $\operatorname{Pr}\left(\hat{x}_{k}^{j}=x_{k}^{i}\right)=\omega_{k}^{i}$. Although there are variants of the resampling algorithm, we use multinomial resampling [21] in this paper because of its simplicity and effectiveness. Therefore, on condition that the total number of particles remains to $N$, particles with high weights will be duplicated several times and are assigned with equal weights $1 / N$.

The resampling step does reduce the effects of the degeneracy problem, but it also has a drawback: the particles with high weights are duplicated several times after resampling, and this would result in a loss of diversity. To overcome such a drawback, an additional step has been introduced, called Metropolis-Hastings (MH) algorithm [10], [20], which is a Markov chain Monte Carlo procedure for generating a sequence of samples from probability distribution. By choosing the probability distribution $\pi(x)$, the algorithm is implemented by the following steps:

1) Generate the rand data from uniform distribution $[0,1]$, $\nu \sim U[0,1]$ 
2) Sample $x_{k}^{*}$ from the proposal density, $x_{k}^{*} \sim q\left(x_{k}^{*} \mid x_{k}^{(i)}\right)$ 3) If $\nu \leq \min \left\{1, \frac{\pi\left(x_{k}^{*}\right) q\left(x_{k}^{(i)} \mid x_{k}^{*}\right)}{\pi\left(x_{k}^{(i)}\right) q\left(x_{k}^{*} \mid x_{k}^{(i)}\right)}\right\}$, accept $x_{k}^{*}$; else reject
$x_{k}^{*}$.

\section{Kernel Smoothing for Parameters Estimation}

In this paper, the Kernel smoothing method is used to estimate the unknown parameters of the system. As discussed in [2], [5], [22], by using the Kernel smoothing method, the problems of covariance increase and performance degeneration could be avoided when using the strategy of adding the parameters as part of the state space vector. Furthermore, this method can deal with both the fixed and time-varying parameters conveniently by tuning the smoothing factor. For this method, the true posterior distribution is represented by

$$
p\left(\theta_{k} \mid z_{1: k-1}\right) \approx \sum_{i=1}^{N} \omega_{k-1}^{i} \kappa\left(\theta_{k} \mid m_{k-1}^{i}, \sigma_{\theta, k-1}^{2}\right)
$$

where $m_{k-1}^{i}$ and $\sigma_{\theta, k-1}^{2}$ are the mean and covariance of the kernel distribution $\kappa\left(\theta_{k} \mid m_{k-1}^{i}, \sigma_{\theta, k-1}^{2}\right)$, respectively. Furthermore, to ensure that the sampling points at time $k$ and $k-1$ have the same mean and variance, a shrinkage method is applied as follows:

$$
\begin{aligned}
m_{k-1}^{i} & =\left(\sqrt{1-h^{2}}\right) \theta_{k-1}^{i}+\left(1-\sqrt{1-h^{2}}\right) \hat{\theta}_{k-1} \\
\sigma_{\theta, k-1}^{2} & =h^{2} \sum_{i=1}^{N}\left(\theta_{t-1}^{i}-\hat{\theta}_{k-1}\right)^{2} \omega_{k-1}^{i}
\end{aligned}
$$

where $\hat{\theta}_{k-1}=\sum_{i=1}^{N} \theta_{t-1}^{i} \omega_{k-1}^{i}$ is the mean of parameters at time $k-1$ and $h$ is the smoothing factor. If the parameters to be estimated are known a prior to be time-invariant, $h$ should be set to a small positive value (e.g. $0<h<0.2$ ). On the other hand, if the parameters are expected to be time-varying, $h$ should take a value close to 1 .

To summarize the above discussion, the pseudo code of particle filter algorithm incorporating kernel smoothing for state and parameter estimation in this paper is described as follows:

1) For $i=1: N$

Draw $\theta_{k}^{i}$ from Gaussian distribution $\kappa\left(\theta_{k} \mid m_{k-1}^{i}, \sigma_{\theta, k-1}^{2}\right)$

Draw $x_{k}^{i}$ from importance density based extended Kalman filter

Assign weight $\hat{\omega}_{k}^{i}$

End

2) Calculate total weight: $t=\operatorname{SUM}\left[\left\{\hat{\omega}_{k}^{i}\right\}_{i=1}^{N}\right]$

3) For $i=1: N$ End

$$
\text { Normalize: } \omega_{k}^{i}=\frac{\hat{\omega}_{k}^{i}}{\operatorname{SUM}\left[\left\{\hat{\omega}_{k}^{i}\right\}_{i=1}^{N}\right]}
$$

4) Resample procedure

5) Metropolis-Hastings algorithm

\section{EXPERIMENTAL RESUlts AND Discussion}

In this section, the particle filtering algorithm combined with the kernel smoothing method is applied to jointly estimate parameters and state variables via the short time series data used in the Ref. [34] and shown in Fig. 2. The upper part of Fig. 2 shows the gold immunochromatographic strip's nitrocellulose membrane pixel intensity inverse the sample flow direction. The images are acquired when the sample passes through the nitrocellulose membrane that consists of 45 equally spaced time points (from 0 to 11 minutes), 45 images as a time series. We choose $z=\left[\max _{\text {pixel }}\left(255-I_{\text {pixel }}\right)-P_{\text {base }}\right] / 10$ as the observed value shown in the lower part of Fig. 2, where $I_{\text {pixel }}$ is the pixel intensity and $P_{\text {base }}$ equals to $\max _{\text {pixel }}\left(255-I_{\text {pixel }}\right)$ of the first out of the 45 images.
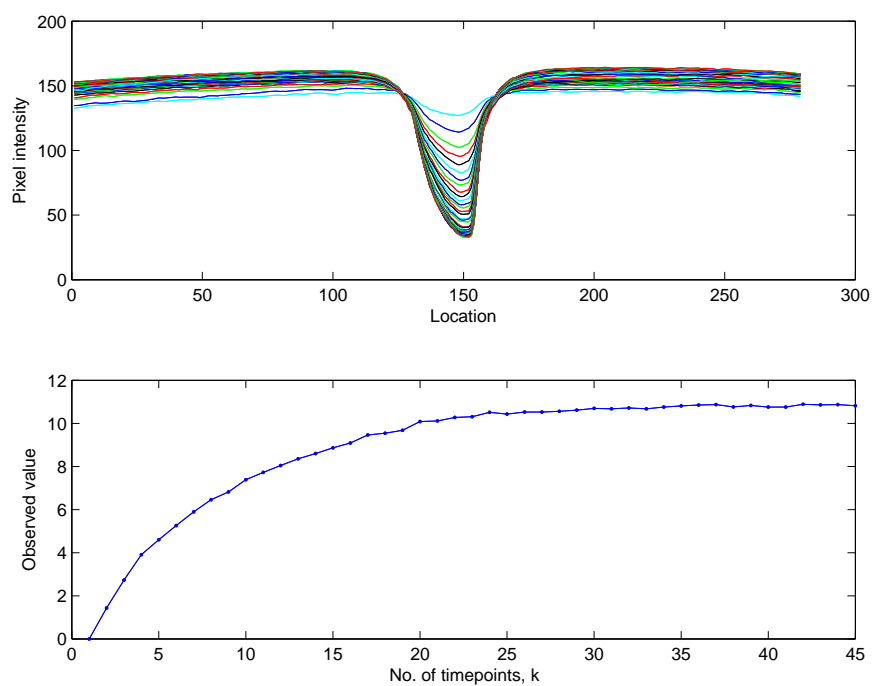

Fig. 2. Upper part: The strip's nitrocellulose membrane pixel intensity inverse the sample flow direction; Lower part: The observed value of lateral flow immunoassay biochemical reaction signal

Take

$x_{0}=[5,6.5,0,13,0,0]^{T}$,

$k_{0}=[0.03,0.0001,0.01,0.0001,0.04,0.0001,0.04,0.0001,2.2]^{T}$

as the initial values of the state variables and parameters, respectively. Furthermore, the positivity constraints on the system states are considered in this paper to ensure that the results are more practical in the real world. Both the identified parameters and state variables are shown in Fig. 3 and Fig. 4, which are depicted in the form of time series.

Furthermore, to evaluate the model quality in a quantitative way, let us introduce the following criterion for the modeling errors (error ratio in percentage) between the actual and the model predicted data [25], [32]:

$$
\text { Error ratio }=100 \times \frac{1}{l} \sum_{c=1}^{l}\left[\sqrt{\frac{\sum_{k=1}^{s}\left(y_{c k}-\hat{y}_{c k}\right)^{2}}{\sum_{k=1}^{s}\left(y_{c k}\right)^{2}}}\right] \%
$$

where $l$ is the number of observations (dimension) involved in the modeling ( $l=1$ in this paper), $s$ is the number of observations (length), and $y_{c k}$ is the actual value for $c$ th observation at the $k$ th time point. It is calculated that the error 

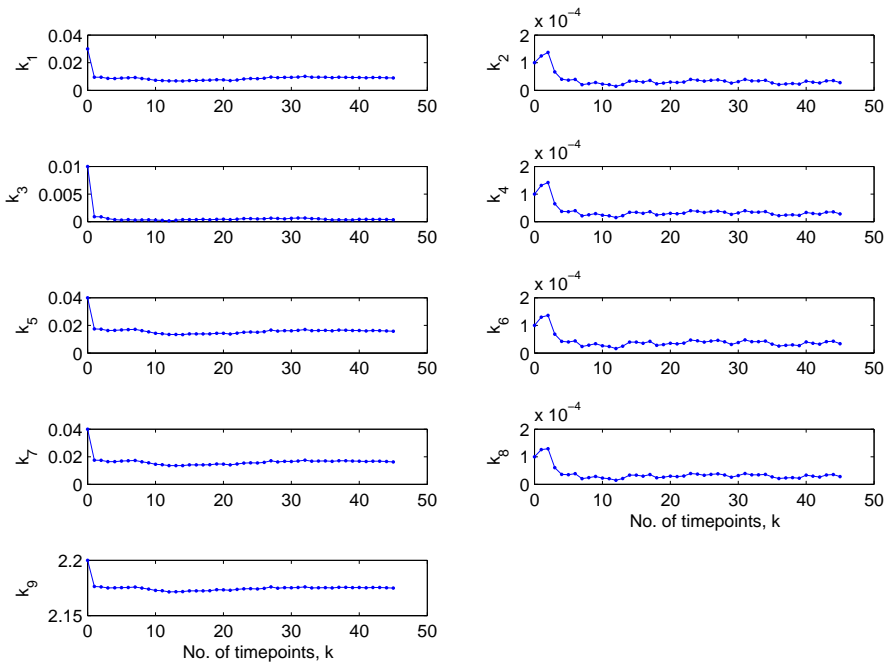

Fig. 3. The estimated time series of parameters $k_{1}, k_{2}, k_{3}, k_{4}, k_{5}$, $k_{6}, k_{7}, k_{8}, k_{9}$.

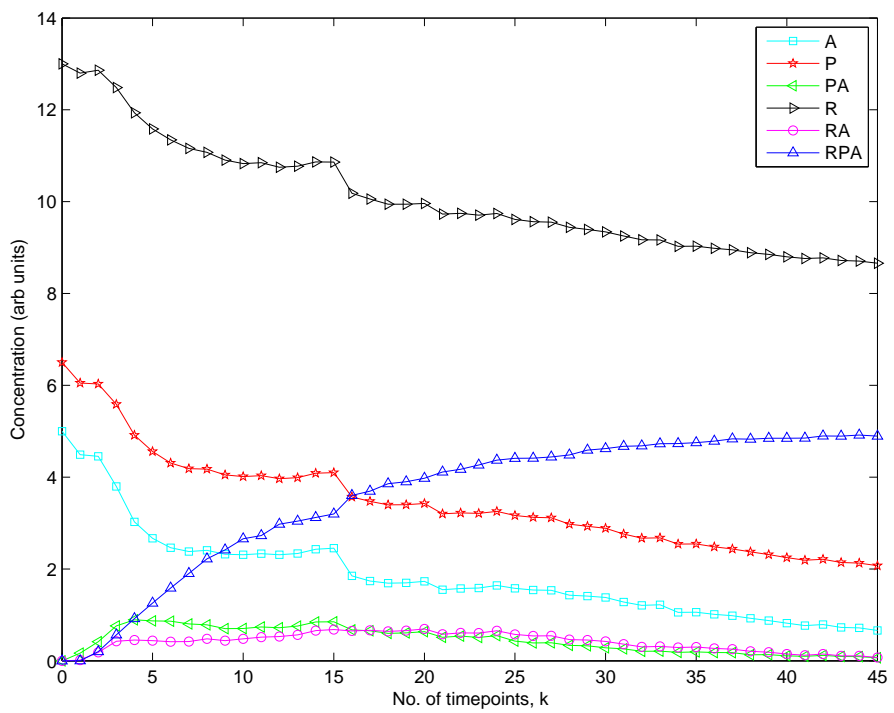

Fig. 4. The estimated time series of states $A, P, P A, R, R A, R P A$.

ratio of the proposed algorithm is $1.14 \%$ through only 45 time points (images), which indicates that the identified model is generally satisfactory.

\section{CONCLUSIONS}

In this paper, we have presented a particle filtering algorithm by incorporating the kernel smoothing method in order to model the lateral flow immunoassay state space model through available but short time-series measurement. The extended Kalman filter has been chosen as the importance density of the particle filter and the Metropolis-Hastings algorithm has been introduced after the resampling step to keep the particles' diversity. Then, the proposed algorithm has been successfully applied to identify the parameters and states of the sandwichtype lateral flow immunoassay model simultaneously. The simulation results has demonstrated the effectiveness of the proposed method.

\section{REFERENCES}

[1] R. Tanaka, T. Yuhi, N. Nagatani, T. Endo, K. Kerman and Y. Takamura, A novel enhancement assay for immunochromatographic test strips using gold nanoparticles, Anal Bioanal Chem, vol. 385, no. 8, pp. 1414-1420, 2006.

[2] J. Liu, M. West, Combined parameter and state estimation in simulationbased filtering, in: A. Doucet, N. de Freitas, N. Gordon (Eds.), Sequential Monte Carlo in Practice, Springer-Verlag, New York, pp. 197-223, 2001.

[3] J. Kaur, K. Singh, R. Boro, K. Thampi, M. Raje and G. Varshney, Immunochromatographic dipstick assay format using gold nanoparticles labeled protein-hapten conjugate for the detection of atrazine, Environmental Science and Technology, vol. 41, no. 14, pp. 5028-5036, 2007.

[4] M. Du, Z. Fang and H. Fei, Application of photoelectric sensor to quantitative determination of immunochro-matographic assay strip, Chinese Journal of Scientific Instrument, vol. 36, no. 7, pp. 671-673, 2005.

[5] T. Chen, J. Morris, E. Martin, Particle filters for state and parameter estimation in batch processes, Journal of Process Control, vol. 15, pp. 665-673, 2005.

[6] K. Faulstich, R. Gruler, M. Eberhard and K. Haberstroh, Developing rapid mobile POC systems. Part 1:Devices and applications for lateralflow immunodiagnostics, IVD Technology, vol. 13, no. 6, pp. 47-53, 2007.

[7] B. N. M. Laska, M. Bolic, R. A. Goubran, Particle filter enhancement of speech spectral amplitudes, IEEE Trans. Audio, Speech, and Language Processing, vol. 18, no. 8, pp. 2155-2167, 2010.

[8] L. Huang, Y. Zhang, C. Xie, J. Qu, H. Huang and X. Wang, Research of reflectance photometer based on optical absorption, International Journal for Light and Electron Optics, vol. 121, no. 19, pp. 1725-1728, 2010.

[9] D. Li, S. Wei, H. Yang, Y. Li and A. Deng, A sensitive immunochromatographic assay using colloidal gold-antibody probe for rapid detection of pharmaceutical indomethacin in water samples, Biosensors and Bioelectronics, vol. 24, no. 7, pp. 2277-2280, 2009.

[10] M. Arulampalam, S. Maskell, N. Gordon, T. Clapp, A tutorial on particle filters for on-line non-linear/non-gaussian bayesian tracking, IEEE Trans. Signal Processing, vol. 50, no. 2, pp. 174-188, 2002.

[11] J. Li, A. Ouellette, L. Giovangrandi, D. Cooper, A. Ricco and G. Kovacs, Optical scanner for immunoassays with up-converting phosphorescent labels, IEEE Trans. Biomedical Engineering, vol. 55, no. 5, pp. 15601571, 2008.

[12] J. Gantelius, C. Hamsten, M. Neiman, J. M. Schwenk, A. Persson, H. Andersson-Svahn, A lateral flow protein microarray for rapid determination of contagious bovine pleuropneumonia status in bovine serum, Journal of Microbiol Methods, vol. 82, no. 1, pp. 11-18, 2010.

[13] C. Mott, G. Dumont, D. B. Boivin, D. Mollicone, Model-based human circadian phase estimation using a particle filter, IEEE Trans Biomedical Engineering, vol. 58, no. 5, pp. 1325-1336, 2011.

[14] Y. Li, N. Zeng and M. Du, Study on the methodology of quantitative gold immunochromatographic strip assay, Proc. 2010 International Workshop on Intelligent Systems and Application, pp. 182-185, 2010.

[15] C. Berzuini, N. G. Best, W. R. Gilks C. Larazza, Dynamic conditional independence models and Markov chain Monte Carlo methods, Journal of the American Statistical Association, vol. 92, pp. 1403-1411, 1997.

[16] D. Hou, F. Fu, A dual particle filter for state and parameter estimation in nonlinear system, Chinese Journal of Electronics and Information Technology, vol. 30, no. 9, pp. 2128-2133, 2008.

[17] C. Robert, G. Casella, Monte carlo statistical methods, Springer, New York, 1999.

[18] A. Doucet, S. Godsill, C. Andrieu, On sequential Monte Carlo sampling methods for Bayesian filtering, Statistics and ComPuting, vol. 10, no. 3 , pp. 197-208, 2000.

[19] A. F. M. Smith, A. E. Gelfand, Bayesian statistics without tears: a sampling-resampling perspective, American Statistician, vol. 46, pp. 8488, 1992.

[20] B. P. Carlin, N. G. Polson, D. S. Stoffer, A Monte Carlo approach to nonnormal and nonlinear state-space modeling, Journal of the American Statistical Association, vol. 87, no. 418, pp. 493-500, 1992.

[21] N. J. Gordon, D. J. Salmond, and A. F. M. Smith, Novel approach to nonlinear/non-Gaussian Bayesian state estimation, IEE Proceedings- $F$, vol. 140 , no. 2 , pp. 107-113, 1993

[22] A. Doucet, V. Tadic, Parameter estimation in general state-space models using particle methods, Annals of the Institute of Statistical Mathematics, vol. 55, pp. 409-422, 2003.

[23] C. An, T. Yoshiki, G. Lee, Y. Okada, Evaluation of a rapid qualitative prostate specific antigen assay, the One Step PSA ${ }^{T M}$ test, Cancer Lett, vol. 162 , no. 2 , pp. $135-139,2001$. 
[24] http://commons.wikimedia.org/wiki/File:Lateral_Flow_Assay.jpg

[25] L. Ljung, System Identification: Theory for the User, 2nd ed. Upper Saddle River, NJ: Prentice-Hall, 1999.

[26] S. Qian and H. Haim, A mathematical model of lateral flow bioreactions applied to sandwich assays, Analytical Biochemistry, vol. 322, no. 1, pp. 89-98, 2003.

[27] S. Qian and H. Haim, Analysis of lateral flow biodetectors: competitive format, Analytical Biochemistry, vol. 326, no. 2, pp. 211-224, 2004.

[28] C. Raphael and Y. Harley, Lateral flow immunoassay, Humana Press, 2008.

[29] A. Mohamed, K. Schwarz, Adaptive Kalman Filtering for INS/GPS, Journal of Geodesy, vol. 73, no. 4, pp. 193-203, 1999.

[30] X. Sun, L. Jin and M. Xiong, Extended Kalman filter for estimation of parameters in nonlinear state-space models of biochemical networks, PLoS ONE, vol. 3, no. 11, e3758, 2008.

[31] Z. Wang, X. Liu, Y. Liu, J. Liang and V. Vinciotti, An extended Kalman filtering approach to modeling nonlinear dynamic gene regulatory networks via short gene expression time series, IEEE/ACM Trans. Computional Biology and Bioformatics, vol. 6, no. 3, pp. 410-419, 2009.

[32] Z. Wang, F. Yang, D. W. C. Ho, S. Swift, A. Tucker and X. Liu, Stochastic dynamic modeling of short gene expression time series data, IEEE Trans. NanoBioscience, vol. 7, no. 1, pp. 44-55, 2008.

[33] P. Yager, T. Edwards, E. Fu, K. Helton, K. Nelson, M. R. Tam, B. H. Weigl, Microfuidic diagnostic technologies for global public health, Nature vol. 442, pp. 412-418, 2006.

[34] N. Zeng, Z. Wang, Y. Li, M. Du and X. Liu, Inference of nonlinear state-space models for sandwich-type lateral flow immunoassay using extended Kalman filtering, IEEE Trans. Biomedical Engineering, vol. 58 , no. 7 , pp. 1959-1966, 2011

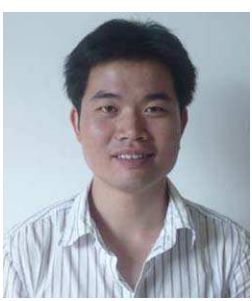

Nianyin Zeng was born in Fujian Province, China, in 1986. He received the B.S. degree in Electrical Engineering and Automation from Fuzhou University, Fuzhou, China, in 2008, where he is currently working toward the Ph.D. degree in Electrical Engineering.

$\mathrm{He}$ is the author or coauthor of several technical papers and also a very active reviewer for many international journals. His current research interests include nonlinear filtering, time-series modeling, and bioinformatics.

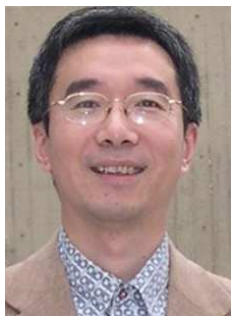

Zidong Wang was born in Jiangsu, China, in 1966. $\mathrm{He}$ received his B.Sc. degree in Mathematics in 1986 from Suzhou University, Suzhou, China, the M.Sc. degree in Applied Mathematics in 1990 and the Ph.D. degree in Electrical and Computer Engineering in 1994, both from Nanjing University of Science and Technology, Nanjing, China.

Dr. Wang is now a Professor of Dynamical Systems and Computing at Brunel University in the UK. His research interests include dynamical systems, signal processing, bioinformatics, control theory and applications. He has published more than 200 papers in refereed international journals.

Dr. Wang is currently serving as an Associate Editor for 12 international journals including IEEE Transactions on Automatic Control, IEEE Transactions on Neural Networks, IEEE Transactions on Signal Processing, IEEE Transactions on Systems, Man, and Cybernetics - Part C and IEEE Transactions on Control Systems Technology.

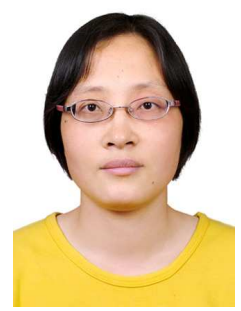

Yurong Li was born in Fujian Province, China, in 1973. She received her Master degree in Industry Automation and Ph.D. in Control Theory and Control Engineering from Zhejiang University, Zhejiang, Chian in 1997 and 2001, respectively.

Now she is an Associate Professor at Fuzhou University. And since 2007, she is the member of Fujian Key Laboratory of Medical Instrumentation \& Pharmaceutical Technology. Her research interests include biomedical instrument and intelligent information processing.

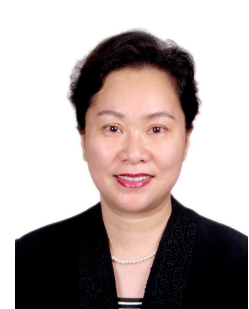

Min Du was born in Fujian Province, China, in 1955. She received her Ph.D. in Electrical Engineering from Fuzhou University, Fuzhou, China, in 2005.

Now she is a professor and doctorial supervisor at Fuzhou University. And since 2007, she is the associate director of Fujian Key Laboratory of Medical Instrumentation \& Pharmaceutical Technology. Her research interests include smart instrument and photoelectrical system.

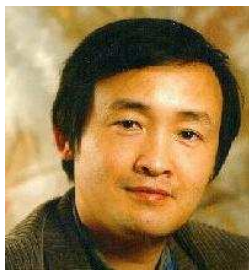

Xiaohui Liu received the B.Eng. degree in computing from Hohai University, Nanjing, China, in 1982 and the Ph.D. degree in computer science from Heriot-Watt University, Edinburg, U.K., in 1988.

$\mathrm{He}$ is currently a Professor of Computing at Brunel University. He leads the Intelligent Data Analysis (IDA) Group, performing interdisciplinary research involving artificial intelligence, dynamic systems, image and signal processing, and statistics, particularly for applications in biology, engineering and medicine. Professor Liu serves on editorial boards of four computing journals, founded the biennial international conference series on IDA in 1995, and has given numerous invited talks in bioinformatics, data mining and statistics conferences. 